\title{
Caracterización clínica de los pacientes con síndrome mielodisplásico
}

\author{
Clinical characterization of patients with \\ myelodysplastic syndrome
}

\author{
Gloria Elena Mora, Daniel Espinosa, Claudia Casas, Virginia Abello, \\ María Helena Solano • Bogota, D.C. (Colombia)
}

\section{Resumen}

Objetivo: describir las características clínicas, sociodemográficas y calidad de vida de los pacientes con síndrome mielodisplasico (MDS).

Diseño: estudio descriptivo de corte transversal.

Marco de referencia: Hospital San José de Bogotá (institución de cuarto nivel).

Población: treinta y nueve pacientes con diagnóstico de MDS, mayores de 18 años, obtenidos en el departamento de estadística.

Mediciones principales: frecuencia de variables clínicas, sociodemográficas y calidad de vida medida por la escala FACT-G.

Resultados: la edad promedio fue de 74 años, con un predominio en hombres. El diagnóstico más frecuente fue MDS tipo displasia mutilinaje, y la citopenia más prevalente fue anemia. La mayoría de los pacientes fue clasificado como muy bajo riesgo o bajo riesgo por la escala IPSS-R y WPSS, y bajo riesgo y riesgo intermedio -1 por la escala IPSS y MDACC. La progresión a leucemia mieloide aguda se presentó en $7.69 \%$, mientras que la mortalidad en $15.38 \%$. Existió una mayor frecuencia de muerte y transformación a leucemia en los pacientes clasificados como de alto riesgo. La calidad de vida fue inferior en los pacientes con anemia y fue superior en el MDS-(del5q).

Conclusiones: las características de la población estudiada son similares a las reportadas en la literatura (predominio en hombres, subtipo de MDS más frecuente, citopenia predominante y distribución de riesgo). Existieron diferencias con respecto a la edad, frecuencia de comorbilidades y su relación con mortalidad. La calidad de vida se correlacionó con el subtipo de MDS y citopenias. (Acta Med Colomb 2016; 41: 36-41).

Palabras clave: síndrome mielodisplásico, calidad de vida, estudio de médula ósea, eritropoyetina, azacitidina.

\footnotetext{
Abstract

Objective: to describe the clinical and socio-demographic characteristics and quality of life of patients with myelodysplastic syndrome (MDS).

Design: a descriptive cross-sectional study.

Framework: Hospital San José de Bogota (fourth level institution).

Population: thirty-nine patients diagnosed with MDS, older than 18 years, obtained in the statistics department.

Main measurements: frequency of clinical and sociodemographic variables and quality of life measured by FACT-G scale.

Results: the mean age was 74 years, with male predominance. The most frequent diagnosis was type mutilineage MDS dysplasia, and the most prevalent cytopenia was anemia. Most patients were classified as very low risk or low risk by IPSS-R and WPSS scale and low-risk and intermediate risk -1by IPSS and MDACC scale. Progression to acute myeloid leukemia occurred in 7.69\%, while mortality occurred in $15.38 \%$. There was a higher incidence of death and transformation to leukemia in patients classified as high risk. The quality of life was lower in patients with anemia and was higher in the MDS-(del5q).
}

Dra. Gloria Elena Mora Figueroa: Residente de Hematología, Fundación Universitaria Ciencias de la Salud; Dres. Daniel Espinosa, Claudia Casas, Virginia Abello y María Helena Solano: Docentes del Servicio de Hematología, Hospital San José. Bogotá, D.C. (Colombia). Grupo de Investigación (GrupLAC): Hematología Hospital de San José - FUCS. Bogotá, D.C. (Colombia)

Correspondencia. Dra. Gloria Elena Mora Figueroa. Bogotá, D.C. (Colombia). E-mail: elenamorafigueroa@gmail.com Recibido 12/XI/2014 Aceptado 16/IV/2015 
Conclusions: The characteristics of the study population are similar to those reported in the literature (predominantly in men, subtype of MDS more frequent, predominant cytopenia and risk distribution). There were differences with respect to age, frequency of comorbidities and their relationship to mortality. Quality of life was correlated with the subtype of MDS and cytopenias. (Acta Med Colomb 2016; 41: 36-41).

Keywords: myelodysplastic syndrome, quality of life, study of bone marrow, erythropoietin, azacytidine.

\section{Introducción}

El síndrome mielodisplásico (MDS) se establece en el año 2008 por la Organización Mundial de la Salud (OMS) (1) como una patología hematológica caracterizada por ser un trastorno clonal, en la cual hay displasia en una o más líneas celulares, hematopoyesis inefectiva, y un aumento en el riesgo de desarrollar leucemia mieloide aguda. En Estados Unidos la incidencia anual es 4.1 casos por 100000 habitantes (2); mientras que en Europa la incidencia oscila entre 2 y 20 casos por 100000 habitantes, siendo más alta en el Reino Unido y más baja en Europa del Este (3). La edad promedio de presentación es 65 años y la forma más frecuente en el MDS es displasia multilinaje (4). El curso natural de la enfermedad es variable, con supervivencias que oscilan entre nueve y 144 meses (4). En Colombia no se cuenta con datos de incidencia ni prevalencia, al igual que se desconoce las particularidades de los pacientes. Teniendo en cuenta que no existe caracterización de los pacientes con MDS en nuestro país, el objetivo del estudio es describir las características clínicas de los pacientes con MDS que asistieron al servicio de hematología en el Hospital de San José en la ciudad de Bogotá, el cual es una institución de cuarto nivel y centro de referencia nacional.

\section{Material y métodos}

Se incluyeron pacientes con diagnóstico de MDS según la definición de la OMS (1) para el año 2008 que asistieron al servicio de hematología del Hospital San José entre el $1^{\circ}$. de enero de 2010 y el 30 de julio de 2014 y se excluyeron menores de 18 años.

Los pacientes fueron seleccionados a partir de los datos suministrados por el departamento de estadística del Hospital San José, y de los que asistieron al servicio consulta de hematología. Los datos sociodemográficos y clínicos fueron obtenidos a partir de la historia clínica.

Se diseñó un formato de recolección de datos en el programa ACCESS $2010^{\circledR}$ para recoger la información correspondiente a variables sociodemográficas y clínicas, y el cual pudiera ser utilizado posteriormente para el seguimiento de los pacientes con MDS en el servicio de hematología. En lo relacionado con las variables sociodemográficas se tuvo en cuenta el estrato socioeconómico y el régimen de afiliación al Sistema General de Salud.

Se evaluaron diversas características clínicas incluyendo la clasificación de riesgo según el International Prognostic Scoring System (IPSS), el Revised International Prognostic
Scoring System (IPSS-R), el WHO classification-based Prognostic Scoring System (WPSS), y la escala de riesgo del MD Anderson Cancer Center (MDAS). De igual manera se documentó el riesgo citogenético, las citopenias presentadas al diagnóstico, el estudio de médula ósea al diagnóstico. Se caracterizó el tratamiento recibido y su respuesta según la definición del grupo internacional de MDS (5).

La calidad de vida fue medida a través de la escala Functional Assessment Cancer Therapy (FACT-G), la cual es una escala de autoaplicación que evalúa cuatro aspectos diferentes incluyendo la situación de salud física general (siete ítems), ambiente familiar y social (siete ítems), estado emocional (seis ítems) y capacidad de funcionamiento personal (siete ítems). Cada ítem se puntúa en una escala tipo Likert de cinco niveles que van desde cero (nada) hasta cuatro (muchísimo), con un puntaje que oscila entre 0 y 108 puntos. A mayor puntaje mayor calidad de vida, sin embargo no existe un punto de corte. Esta escala se encuentra validada en nuestro país a partir del estudio de Sánchez R, et al. (6).

Las variables cualitativas fueron descritas por medio de frecuencias absolutas y relativas. Las variables cuantitativas fueron descritas por medio de medias y desviaciones estándar o mediana y rangos intercuartílicos dependiendo de la forma de la distribución de los datos. El puntaje de calidad de vida fue analizado de acuerdo con la clasificación de riesgo de los pacientes según la escala WPSS, según la clasificación del MDS y las citopenias presentadas al diagnóstico. Estos análisis fueron realizados en el paquete estadístico STATA $12^{\circledR}$. El trabajo fue aprobado por el Comité de Ética en investigación en humanos del Hospital San José.

\section{Resultados}

Se encontraron 39 pacientes con MDS que cumplieron con los criterios de inclusión y exclusión, con un promedio de edad de 74 años y con un predominio de hombres en la población analizada (relación 2:1) (Tabla 1). La mayoría de los pacientes eran de estrato 1 y 2 , y correspondían a régimen contributivo, esta asociación se encuentra en relación con la población atendida en el Hospital San José.

El $43.6 \%$ de los pacientes tenían displasia multilinaje (RCMD) y $33.3 \%$ presentaban anemia refractaria con exceso de blastos tipo 1 o 2, siendo la mayoría tipo 2. Ninguno de los pacientes tuvo MDS secundario a tratamiento. La citopenia más frecuentemente presentada fue anemia, con un nivel de hemoglobina promedio en $10.39 \mathrm{mg} / \mathrm{dL}$ (DE 
Tabla 1. Características sociodemográficas (n:39).

\begin{tabular}{|c|c|}
\hline \multicolumn{2}{|c|}{ Variables sociodemográficas } \\
\hline Edad (media-desviación) & $74.25(9.02)$ \\
\hline Sexo (n-\%) & \\
Hombres & $24(62.5)$ \\
Mujeres & $15(38.46)$ \\
\hline Diagnóstico (n-\%) & \\
RCMD & $17(43.59)$ \\
RCUD & $5(12.82)$ \\
RAEB-1 & $5(12.82)$ \\
RAEB-2 & $8(20.51)$ \\
MDS -(del5q) & $2(5.13)$ \\
MDS-UC & $2(5.13)$ \\
RARS & $0(0)$ \\
\hline
\end{tabular}

3.21), seguida de leucopenia, con un promedio de recuento absoluto de neutrófilos de $1.540 \mathrm{cel} / \mathrm{uL}$ (DE 1.242). Este dato se encuentra en concordancia con el encontrado en el requerimiento transfusional, de tal manera que el promedio de unidades transfundidas de glóbulos rojos cada mes fue 0.86 unidades. De igual manera se encontró que el promedio de ferritina fue de $1.080 \mathrm{ng} / \mathrm{mL}$; sin embargo, tan sólo dos pacientes se encontraban en manejo con terapia quelante.

El tiempo transcurrido desde el inicio de los síntomas hasta el diagnóstico fue en promedio 90 días (rango intercuartílico entre 28 y 457 días). El $60.53 \%$ de los pacientes presentaban alguna comorbilidad, encontrando patologías cardiovasculares en más del $90 \%$ de los pacientes (Tabla 2).

En el estudio de médula ósea al diagnóstico, la displasia más frecuentemente encontrada fue la displasia eritroide, seguida de la megacariocítica. La variante hipoplásica se encontró en $7.79 \%$ de los pacientes y el promedio de blastos fue $4.48 \%$.

En cuanto a las escalas de riesgo, la mayoría de los pacientes correspondían a riesgo bajo e intermedio -1 según el IPSS y MDACC, muy bajo riego y bajo riesgo según el IPSS-R y WPSS. Se contó con cariotipo en 29 pacientes, predominando el cariotipo normal en $73.3 \%$ de los pacientes, resaltándose que el promedio de metafases analizadas fue de 20. La anomalía genética más frecuente fue la deleción del $5 q$; de tal manera que el riesgo citogenético correspondió en $95 \%$ a muy bueno y bueno (Tabla 3 ).

La quimioterapia más utilizada fue la 5- azacitidina, seguida de lenalidomida y decitabine (Tabla 4). De igual manera la terapia de soporte más prescrita fue la eritropoyetina en $48 \%$ de los pacientes. Dentro de los pacientes manejados con EPO se logró una respuesta eritroide en $47 \%$, resaltándose que $53 \%$ de los pacientes con dosis menores a 30 000u semanales lograron esta respuesta. En los pacientes con 5-AZA no se logró respuesta hematológica, y se presentó progresión de la enfermedad en $42.8 \%$ de los pacientes y la enfermedad permaneció estable en $28.5 \%$ de los pacientes.
Tabla 2. Comorbilidades.

\begin{tabular}{|c|l|}
\hline Comorbilidades conocidas (n-\%) & \\
$\mathrm{Si}$ & $23(60.53)$ \\
No & $12(31.58)$ \\
Desconocido & $3(7.89)$ \\
\hline Pacientes con comorbilidades conocidas (n:23) & \\
Diabetes mellitus & $7(30.34)$ \\
Cardiovascular & $21(91.3)$ \\
Enfermedad renal crónica & $4(17.39)$ \\
\hline
\end{tabular}

Tabla 3. Clasificación de riesgo $n$ (\%).

\begin{tabular}{|c|c|}
\hline \multicolumn{2}{|l|}{ IPSS (n:28) } \\
\hline Bajo riesgo & $9(32.14)$ \\
\hline Intermedio - 1 & $16(57.14)$ \\
\hline Intermedio-2 & $3(10.71)$ \\
\hline Alto riesgo & $0(0)$ \\
\hline \multicolumn{2}{|l|}{ IPSS-R (n:28) } \\
\hline Muy bajo riesgo & $3(10.71)$ \\
\hline Bajo riesgo & $14(50)$ \\
\hline Riesgo intermedio & $8(28.57)$ \\
\hline Alto riesgo & $3(10.71)$ \\
\hline Muy alto riesgo & $0(0)$ \\
\hline \multicolumn{2}{|l|}{ WPSS (n: 29) } \\
\hline Muy bajo riesgo & $3(10.32)$ \\
\hline Bajo riesgo & $13(44.82)$ \\
\hline Riesgo intermedio & $4(13.79)$ \\
\hline Alto riesgo & $9(31.03)$ \\
\hline Muy alto riesgo & $0(0)$ \\
\hline \multicolumn{2}{|l|}{$\operatorname{MDACC}(\mathrm{n}: 28)$} \\
\hline Bajo riesgo & $9(32.14)$ \\
\hline Riesgo intermedio -1 & $9(32.14)$ \\
\hline Riesgo intermedio -2 & $1(3.57)$ \\
\hline Alto riesgo & $4(14.29)$ \\
\hline \multicolumn{2}{|l|}{ Cariotipo (n:30) } \\
\hline Sin crecimiento & $1(3.33)$ \\
\hline $46 \mathrm{XX}$ & $12(40)$ \\
\hline $46 X Y$ & $10(33.33)$ \\
\hline $46 X X \operatorname{del}(5 q) / 46 X Y \operatorname{del}(5 q)$ & $2(6.66)$ \\
\hline $46 X X$ inv(9) del $(5 q)$ & $1(3.33)$ \\
\hline $46 \operatorname{del}(6 \mathrm{p} 12) \operatorname{der}(1212 \mathrm{p} 11.2) / 46 \mathrm{XX}$ & $1(3.33)$ \\
\hline $46 \times X / 46 X X \operatorname{del}(5 q)$ & $1(3.33)$ \\
\hline $46 X Y / 46 X Y \operatorname{del}(11 q)^{\circ}$ & $1(3.33)$ \\
\hline Metafases analizadas (media-desviación) & $20(18.06)$ \\
\hline \multicolumn{2}{|l|}{ Riesgo citogenético (n:29) } \\
\hline Muy bueno & $2(6,89)$ \\
\hline Bueno & $26(89,65)$ \\
\hline Intermedio & $0(0)$ \\
\hline Malo & $1(3,44)$ \\
\hline Muy malo & $0(0)$ \\
\hline
\end{tabular}


Tabla 4. Tratamiento.

\begin{tabular}{|c|c|}
\hline Tratamiento (n- \%) & \\
5- AZA & $7(17.95)$ \\
Decitabina & $1(2.57)$ \\
Lenalidomida & $2(5.13)$ \\
Poliquimioterapia & $1(2.57)$ \\
EPO & $19(48)$ \\
G-CSF & $2(5.13)$ \\
EPO n:21 & \\
& \\
Respuesta eritroide (n-\%) & $10(47.62)$ \\
Según dosis & $8(53.3)$ \\
$25000-30000 \mathrm{u}(\mathrm{n}-\%)$ & $2(50)$ \\
\hline $50000 \mathrm{u}(\mathrm{n}-\%)$ & \\
\hline
\end{tabular}

Existió una progresión a leucemia mieloide aguda en $7.69 \%$, mientras que la mortalidad fue $15.38 \%$. Todos los pacientes que progresaron a leucemia mieloide aguda fallecieron, y dos de las tres muertes adicionales se relacionaron con procesos infecciosos. La mortalidad y la progresión a leucemia se presentó en los pacientes que fueron clasificados como alto riesgo según la WPSS y los del grupo de riesgo intermedio y alto riesgo según el IPSS-R. Mientras que dichos desenlaces se presentaron en los pacientes con alto riesgo, intermedio- 2 e intermedio - 1 según MDACC e IPSS. Los pacientes fallecidos y con progresión a leucemia tenían un recuento de plaquetas y de niveles de hemoglobina menores que los que no presentaron estas complicaciones, lo contrario se observó en el porcentaje de blastos en médula ósea al diagnóstico (Tabla 5).
Tabla 6. Calidad de vida.

\begin{tabular}{|ll|}
\hline $\begin{array}{l}\text { T general (media-desviación) } \\
\text { Mínimo - máximo }\end{array}$ & $\begin{array}{l}54(10.94) \\
37-70\end{array}$ \\
\hline $\begin{array}{ll}\text { Estado físico general de salud (media-desviación) } \\
\text { Mínimo - máximo }\end{array}$ & $\begin{array}{l}12.8(5.84) \\
\text { mar-21 }\end{array}$ \\
\hline Ambiente familiar y social (media-desviación) & $18.6(4.52)$ \\
Mínimo - máximo & $10-25$ \\
\hline Estado emocional (media-desviación) & $13.8(4.02)$ \\
Mínimo - máximo & $7-21$ \\
\hline Capacidad de Funcionamiento individual (media-desviación) & $9.9(6.02)$ \\
Mínimo - máximo & $3-23$ \\
\hline
\end{tabular}

El puntaje promedio en la escala FACT-G fue de 54 puntos, lográndose mayores puntajes en las áreas de ambiente familiar y social y en el estado emocional (Tabla 6). Los pacientes con diagnóstico de MDS -(del5q) tuvieron un puntaje global mayor que los pacientes con otros subtipos de MDS. De igual manera los pacientes con menores niveles de hemoglobina al diagnóstico presentaban un menor puntaje en la escala global. La escala FACT-G fue aplicada a 10 pacientes, lo que no permite llegar a una significancia estadística.

\section{Discusión}

Dado la ausencia de datos a nivel nacional, el presente estudio caracterizó un subgrupo de pacientes con MDS en Colombia, encontrando una edad promedio al diagnóstico

Tabla 5. Niveles de citopenias en la muestra de participantes que fallecieron o progresaron a LMA.

\begin{tabular}{|c|c|c|c|c|}
\hline Grupos & Hemoglobina al diagnóstico & Plaquetas al diagnóstico & RAN al diagnóstico & $\%$ de blastos en m. ósea \\
\hline \multicolumn{5}{|c|}{ Sin Progresión a leucemia mieloide aguda (LMA) } \\
\hline Mediana & 10.5 & 189000 & 1143.5 & 2 \\
\hline $\operatorname{Min}-\max$ & $4-18.7$ & 2000- 687000 & $53-4590$ & $0-16$ \\
\hline $25-75 \%$ & $7.8-13$ & $80000-265000$ & $600-2135$ & $0-9$ \\
\hline \multicolumn{5}{|c|}{ Con progresión a LMA } \\
\hline Mediana & 9.4 & 100000 & 830 & 7.6 \\
\hline Min - max & $7.5-9.8$ & $9000-353000$ & $200-3350$ & abr-13 \\
\hline $25-75 \%$ & $7.5-9.8$ & $9000-353000$ & $200-3350$ & abr-13 \\
\hline \multicolumn{5}{|l|}{ Fallecidos } \\
\hline Mediana & 5.8 & 62500 & 1650 & 8.55 \\
\hline Min - max & $4-9.4$ & $2000-559000$ & $53-4300$ & $0-16$ \\
\hline $25-75 \%$ & $7.5-9.1$ & $9000-353000$ & $200-3350$ & $2-11.8$ \\
\hline \multicolumn{5}{|l|}{ No fallecidos } \\
\hline Mediana & 11.2 & 189000 & 1087 & 2 \\
\hline Min $-\max$ & $6-18.7$ & $19000-687000$ & $289-4590$ & $0-15$ \\
\hline $25-75 \%$ & $7.8-13.1$ & $99000-264000$ & $600-1810$ & $0-9$ \\
\hline
\end{tabular}


superior a la encontrada en Estado Unidos (75 años contra 65 años). Esta diferencia de edad puede estar en relación con un mayor número de pacientes diagnosticados asintomáticos en países desarrollados, al igual que una mayor demora entre el inicio de los síntomas y el diagnóstico en nuestro país. En nuestro estudio se encontró que el tiempo transcurrido entre el inicio de los síntomas y el diagnóstico fue en promedio de 90 días, sin contarse con reportes en la literatura sobre esta estadística en otros países. La educación a los pacientes, una sensibilización del equipo médico sobre esta patología y un mayor acceso a los servicios de salud al facilitar los trámites administrativos podrían disminuir este tiempo.

No existió diferencia en otras características clínicas con lo reportado en otros estudios en Estados Unidos (2) como en el predominio de hombres, el subtipo histológico más frecuente, y la citopenia más prevalente al diagnóstico. La coloración de hierro se realizó en menos de $10 \%$ de los estudios de médula ósea, lo que puede disminuir el diagnóstico de MDS con sideroblastos en anillo. De igual manera tampoco existieron diferencias con respecto a la distribución en los diferentes grupos de riesgo según lo determinado por la escala IPSS e IPSS-R.

En la mayoría de los pacientes evaluados se contó con cariotipo, lográndose un análisis de un número significativo de metafases. Se ha descrito en diferentes revisiones de la literatura (7) una frecuencia de $\operatorname{del}(5 q)$ en $5 \%$, en nuestro estudio dicha anomalía fue $6.6 \%$.

Como hallazgo interesante, se presentó una alta frecuencia de comorbilidades incluyendo las patologías cardiovasculares (enfermedad coronaria, enfermedad arterial periférica y accidente cerebrovascular). Este hallazgo puede estar en relación con la edad promedio de la población estudiada. De los pacientes fallecidos, $66 \%$ tenían alguna comorbilidad, siendo la enfermedad renal crónica la más frecuentemente encontrada (83\%). Se ha descrito una mayor muerte en los pacientes con EPOC y falla cardiaca que en otro tipo de enfermedades (8), lo cual no se observó en nuestro trabajo.

Teniendo en cuenta la alta frecuencia de anemia y de requerimiento transfusional, se evidenció que la ferritina promedio de los pacientes fue superior a $1000 \mathrm{ng} / \mathrm{mL}$; sin embargo, tan sólo dos pacientes se encontraban en manejo con terapia quelante, lo que no permitió hacer una correlación con la calidad de vida u otros desenlaces como frecuencia de comorbilidades y muerte.

En lo relacionado a la respuesta alcanzada con las medidas de soporte, se describió una respuesta a EPO en $47.6 \%$, lo que es concordante con lo encontrado (43.9\%) en el metanálisis realizado por Moyo V, et al. (9). La dosis utilizada menor a $30000 \mathrm{u} / \mathrm{semana}$ tuvo una respuesta de $50 \%$. En nuestro país, existen trabas administrativas para la autorización de dosis superiores a $30000 \mathrm{u} / \mathrm{semana}$, y esto conlleva a que se utilicen dosis inferiores a las recomendadas por las guías internacionales.

En los pacientes que recibieron 5-AZA no se logró respuesta hematológica, resaltándose el mataanálisis realizado por Gurion R, et al (10) que no mostró independencia transfusional. Los demás esquemas de manejo se administraron en un número reducido de pacientes, lo que limita análisis de la respuesta alcanzada.

El porcentaje de mortalidad duplicó el porcentaje de transformación a leucemia mieloide aguda. La discrepancia entre estos dos desenlaces está relacionada con las complicaciones asociadas a las citopenias, resaltándose que todos los pacientes que presentaron transformación a LMA fallecieron. Dos casos fallecieron por procesos infecciosos y no se cuenta con la causa de muerte en un caso.

La evaluación de calidad de vida se realizó en 10 pacientes, resaltándose que el número limitado de pacientes no permite realizar análisis en torno al estrato socioeconómico, el régimen de afiliación al sistema general de salud, o el uso de quelantes de hierro. Tal y como se describe en la literatura (11), la calidad de vida se ve disminuida en los pacientes con mayor severidad de la anemia y es superior en los pacientes con buen pronóstico citogenético. El dominio con menor puntaje fue el de capacidad de funcionamiento individual, esto puede estar explicado por la marcada astenia y adinamia que conlleva la enfermedad. En los estudios publicados sobre calidad de vida en MDS suele evaluarse la mejoría luego de la implementación de intervenciones, pero no se presentan los puntajes obtenidos, por lo cual no fue posible comparar el resultado de nuestros pacientes con los de otras investigaciones.

La principal limitación de este estudio se concentra en el número de sujetos evaluados, al igual que los datos incompletos en las historias clínicas. La posibilidad de llevar a cabo estudios de cooperación entre instituciones y entre grupo internacionales, principalmente de Latinoamérica, nos permitirá resolver el número de pacientes analizado. Se diseñó un formato en Access ${ }^{\circledR}$ para ser utilizado en el servicio de hematología del Hospital San José, con el fin de estandarizar los datos consignados en la historia clínica y de esta manera mejorar el problema de los datos incompletos durante las evaluaciones por nuestro servicio.

La principal fortaleza de nuestro estudio es la descripción detallada de las características clínicas de los pacientes con MDS, la cual no ha sido observada en otros estudios, por ejemplo el uso de varias escalas de riesgo, las displasias presentadas en la médula ósea, el tiempo entre el inicio de los síntomas y el diagnóstico. Así pues, se logró una primera aproximación a los pacientes con MDS en nuestro país.

Estudios prospectivos podrían ampliar nuestro conocimiento sobre esta enfermedad, incluyendo el impacto en la calidad de vida con las intervenciones realizadas en estos pacientes, al igual que se podría establecer el valor predictivo de las diferentes escalas de riesgo en nuestros pacientes.

\section{Agradecimientos}

A nuestros pacientes, por ser una inspiración cada día y por su colaboración durante el estudio. 


\section{Declaración de fuentes de financiación y posibles conflictos de interés}

Los autores no declaran tener conflictos de interés, y el estudio no conto con fuentes de financiación.

\section{Referencias}

1. Cancer TIAfRo. WHO Classification of Tumours of Haematopoietic and Lymphoid Tissue (IARC WHO Classification of Tumours). Swerdlow S, Campo E, Harris NL, Jaffe ES, Pileri SA, Stein H, et al. Editors: World Health Organization. 2008 2008-10. 441 p.

2. Ma X, Does M, Raza A, Mayne ST. Myelodysplastic syndromes: incidence and survival in the United States. Cancer. 2007; 109 (8): 1536-42.

3. Aul C, Gattermann N, Schneider W. Age-related incidence and other epidemiological aspects of myelodysplastic syndromes. Br J Haematol. 1992; 82 (2): 358-67.

4. Wang XQ1, Ryder J, Gross SA, Lin G, Irons RD. Prospective analysis of clinical and cytogenetic features of 435 cases of MDS diagnosed using the WHO (2001) classification: a prognostic scoring system for predicting survival in RCMD. Int J Hematol. 2009 Oct; 90 (3): 361-9. Epub 2009 Aug 29.
5. Cheson BD1, Bennett JM, Kantarjian H, Pinto A, Schiffer CA, Nimer SD, et al. Report of an international working group to standardize response criteria for myelodysplastic syndromes. Blood. 2000 Dec 1; 96 (12): 3671-4.

6. Sánchez R, Ballesteros M, Arnold BJ. Validation of the FACT-G scale for evaluating quality of life in cancer patients in Colombia. Qual Life Res. 2011; 20 (1): 19-29

7. Tefferi A, Vardiman JW. Myelodysplastic Syndromes. N Engl J Med. 2009; 361: 1872-1885.

8. Ma X. Epidemiology of myelodysplastic syndromes. Am J Med. 2012 Jul; 125 (7 Suppl): S2-5.

9. Moyo V, Lefebvre P, Duh MS, Yektashenas B, Mundle S. Erythropoiesisstimulating agents in the treatment of anemia in myelodysplastic syndromes: a meta-analysis. Ann Hematol. 2008 Jul; 87 (7): 527-36.

10. Gurion R1, Vidal L, Gafter-Gvili A, Belnik Y, Yeshurun M, Raanani P. 5-azacitidine prolongs overall survival in patients with myelodysplastic syndrome-a systematic review and meta-analysis. Haematologica. 2010 Feb; 95 (2): 303-10.

11. Stasi R, Abruzzese E, Lanzetta G, Terzoli E, Amadori S. Darbepoetin alfa for the treatment of anemic patients with low- and intermediate-1-risk myelodysplastic syndromes. Ann Oncol. 2005; 16 (12): 1921-7. 